\title{
Rapidly photocurable silk fibroin sealant for clinical applications
}

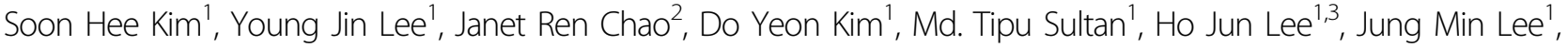 \\ Ji Seung Lee', Ok Joo Lee ${ }^{1}$, Heesun Hong ${ }^{1}$, Hanna Lee ${ }^{1}$, Olatunji Ajiteru', Ye Ji Suh', Hak Soo Choi $\mathbb{1}^{4}$, \\ Yong-Jun Cho ${ }^{5}$ and Chan Hum Park $\mathbb{B}^{1,3}$
}

\begin{abstract}
Sealants are useful as agents that can prevent the leakage of gas or nonclotting fluids from damaged tissues and of blood from the vascular system following injury or repair. Various formulations for sealants have been developed and applied clinically, but problems still remain in terms of biocompatibility issues, long crosslinking times and low adhesive properties. Herein, to address these issues, we report a methacrylated silk fibroin sealant (Sil-MAS) with rapidly crosslinkable, highly adhesive and biocompatible properties and demonstrate its versatility as a medical glue. The excellent physical properties of Sil-MAS are revealed via in vitro mechanical tests and ex vivo aorta pressure tests. In addition, in in vivo biological tests on the skin, liver, and blood vessels of rats, Sil-MAS showed a superb hemostatic and adhesive ability, with high biocompatibility. Specifically, Sil-MAS strongly contributed to faster wound healing than commercially available materials. Furthermore, we showed a successful proof of concept that Sil-MAS could serve as an ideal photocuring laparoscopic medical glue in a laceration rabbit model of liver and stomach serosa using a homemade endoscopic device. These findings on the applicability of rapidly photocurable silk fibroin indicate that SilMAS is a suitable material to supplant existing sealants, adhesives, or hemostatic agents.
\end{abstract}

\section{Introduction}

To stop bleeding, sealing tissues and gluing vessels together have become increasingly important, especially with the expansion of minimally invasive surgery. Medical glues including hemostatic agents, sealants and adhesives have therefore become more critical in surgical practice ${ }^{1}$. The ideal medical glues should be safe, easy to use, low cost and able to meet regulatory requirements in addition to efficacy ${ }^{1}$. Although various products fulfill some of these criteria, issues remain with each of them. Many hemostatic agents are available as powders, sponges, thin films or sheets for surface wounds when pressure must be applied or for covering large areas of profusely bleeding

\footnotetext{
Correspondence: Chan Hum Park (hlpch@paran.com)

${ }^{1}$ Nano-Bio Regenerative Medical Institute, College of Medicine, Hallym

University, Chuncheon 24252, Republic of Korea

${ }^{2}$ School of Medicine, George Washington University, Washington, DC 20037, USA

Full list of author information is available at the end of the article

These authors contributed equally: Soon Hee Kim, Young Jin Lee
}

wounds that have regular shapes. What is needed, however, is a medical glue that could be applied to wounds of various shapes, repair soft tissue and have the capability of being crosslinked once in place. Among the various medical glues, fibrin sealant is the only commercially available material applicable for all three functions, as a hemostatic agent, sealant and adhesive, but the risks of virus transmission and poor adhesion under wet conditions need to be addressed ${ }^{2}$.

Silk fibroin (SF) has been studied extensively due to its high biocompatibility in wound dressings ${ }^{3}$, enzyme immobilization matrixes ${ }^{4}$, tympanic membranes ${ }^{5}$, ridge preservation grafts $^{6}$, and vascular prosthesis and structural implants ${ }^{7,8}$. Additionally, SF has already been developed as a medical glue including SF blended with polyvinyl alcohol and hyaluronic acid ${ }^{9}$, SF hybridized with duck's feet collagen ${ }^{10}$, and RGD peptide-fused recombinant mussel adhesive protein ${ }^{11}$. In this manner, to be applied to medical glue, SF has been fabricated as a solid 
material (e.g., powder, sponge, or mesh) to provide a mechanical tamponade, which limits its application to irregular or large areas. SF-based medical glues have also been developed as colloidal-type glues in a few studies, for example, SF blended with chemically active polyethylene glycol (PEG) ${ }^{12}$ and catechol-functionalized SF containing $\mathrm{PEG}^{13}$. In these studies, SF has been shown to be effective by supporting other materials; in combination, SF can increase the hydrophobicity or mechanical strength or alter the degradability. More recently, tannic acidintroduced $\mathrm{SF}^{14}$ has also been developed; however, this method has a long gelation time. To our knowledge, rapidly crosslinkable colloidal medical glues composed of SF alone have never been studied. Recently, temporally and spatially controlling the physical properties of SF has become available through the photopolymerization of SF methacryloyl ${ }^{15}$. The functionality of SF was improved with SF methacryloyl for fast crosslinking, ease of use (e.g., bioink), and consistent rheological and mechanical properties while maintaining its excellent biocompatibility; therefore, we can now extend the application of SF methacryloyl beyond existing biomedical applications of SF. Herein, we report the applicability of SF methacryloyl as a versatile medical glue that can be applied as a hemostatic agent, sealant, or adhesive. To do so, we systematically evaluated the adhesive properties of SF methacryloyl under various conditions in vitro and its sealing/hemostasis properties via adhesion on skin, liver, and vascular structures in vivo or ex vivo. Finally, laparoscopic surgery was performed with a homemade endoscopic device consisting of SF methacryloyl and a light source in a rabbit liver/stomach laceration model.

\section{Materials and methods Materials}

The photoinitiator lithium phenyl-2,4,6-trimethylbenzoylphosphinate (LAP) was purchased from Tokyo Chemical Industry, Tokyo, Japan. The other reagents including glycidyl methacrylate solution (GMA) were purchased from Sigma-Aldrich (St. Louis, MO, USA). Fibrin glue (Greenplast $\mathrm{Q}^{\circledR}$, Green Cross Corp., Yongin, Korea), polyurethane (Medifoam ${ }^{\circledR}$ liquid bandage: MLB, Mundipharma, Seoul, Korea), Avitene ${ }^{\mathrm{TM}}$ (C. R. Bard, Inc., Murray Hill, NJ, USA) or standard gauze were used as control materials for the various analyses.

\section{Preparation of the silk fibroin sealant (Sil-MAS)}

Sil-MAS was synthesized according to our established protocol ${ }^{15}$. Bombyx mori (B. mori) cocoons were provided by the Rural Development Administration (Jeonju, Korea). Each silkworm cocoon was sliced into four pieces. Forty grams of sliced cocoons were boiled in $1 \mathrm{~L}$ of $0.05 \mathrm{M}$ $\mathrm{Na}_{2} \mathrm{CO}_{3}$ solution for $30 \mathrm{~min}$ at $100^{\circ} \mathrm{C}$ to remove sericins from the silk and then were washed with distilled water
(DW) several times. The degummed silk was dried at room temperature, and $20 \mathrm{~g}$ of it was dissolved in $100 \mathrm{~mL}$ of $9.3 \mathrm{M}$ lithium bromide solution (in DW) at $60^{\circ} \mathrm{C}$ for $1 \mathrm{~h}$ to make regenerated SF solutions. Then, $6 \mathrm{~mL}(424 \mathrm{mM})$ of GMA was slowly added to the mixture with stirring at $300 \mathrm{rpm}$ and $60^{\circ} \mathrm{C}$ for $3 \mathrm{~h}$. Next, the resulting solution was filtered through a rayon-polyester membrane (Miracloth, Calbiochem $^{\circledR}$, San Diego, CA, USA) and dialyzed against DW using $12-14 \mathrm{kDa}$ cutoff dialysis tubes for 7 days. Finally, this methacrylated SF solution was frozen at $-80^{\circ} \mathrm{C}$ for $12 \mathrm{~h}$ and freeze-dried for $48 \mathrm{~h}$. The lyophilized methacrylated SF ( $d$-Sil-MAS) was stored at $-80^{\circ} \mathrm{C}$ for further use.

$d$-Sil-MAS was dissolved in DW at a concentration of $25 \%(\mathrm{w} / \mathrm{v})$. Then, LAP filtered through a cellulose acetate syringe filter $(0.2 \mu \mathrm{m}$, GVS Filter Technology Inc., Findlay, $\mathrm{OH}, \mathrm{USA}$ ) was added into the solution at a concentration of $0.3 \%(\mathrm{w} / \mathrm{v})$ and mixed until fully dissolved using a planetary vacuum mixer (ARV-310, Thinky Corp., Tokyo, Japan). This solution was then strained through a nylon mesh cell strainer $(40 \mu \mathrm{m}$, SPL Co., Pocheon, Korea) and finally pasteurized at $60^{\circ} \mathrm{C}$ for $30 \mathrm{~min}$. For in vitro and ex vivo testing, the sterilization process was skipped. The final solution was called liquid (l)-Sil-MAS. After stabilization at room temperature for $30 \mathrm{~min}$, the $l$-Sil-MAS was irradiated with ultraviolet (UV) light for $10-30 \mathrm{~s}$ at a distance of $5 \mathrm{~cm}$ using a UV spot-curing machine (SPLED-1, ARC UV Corp., Yongin, Korea) equipped with a filter of $365 \mathrm{~nm}$. The spot diameter was $1.5 \mathrm{~cm}$, and the light intensity was measured at $6 \mathrm{~mW} / \mathrm{cm}^{2}$ by a UV meter. This crosslinked material was called $c$-Sil-MAS.

$c$-Sil-MAS samples for the mechanical test and in vivo biocompatibility test were 3D printed by a digital light processing (DLP) printer that was customized by professional manufacturers (NBRTech. Ltd, Chuncheon, Korea) ${ }^{15}$. Samples were designed by CADian3D (IntelliKorea, Seoul, Korea) depending on the test conditions. The following printing parameters were used: printing thickness, $50 \mu \mathrm{m}$; number of base layers, 3; curing time of base layer, $4 \mathrm{~s}$; number of buffer layers, 1 ; and curing time of buffer layers, $3 \mathrm{~s}$. Prints were carried out by repeating the process of projecting an image into the hydrogel followed by raising the Z-stage in an aseptic environment. After printing, the printed objects were rinsed with saline to remove uncrosslinked $l$-Sil-MAS surrounding the $c$-SilMAS before testing.

\section{Characterization of Sil-MAS}

To determine the successful synthesis of the sealant prepolymer, $d$-Sil-MAS was examined through proton nuclear magnetic resonance ( ${ }^{1} \mathrm{H}$ NMR) spectroscopy at a frequency of $400 \mathrm{MHz}$ using a Bruker DPX FT-NMR Spectrometer (9.4 T, Bruker Analytik GmbH, Karlsruhe, Germany). First, $5 \mathrm{mg}$ of $d$-Sil-MAS was dissolved in 
$700 \mu \mathrm{L}$ of deuterium oxide $\left(\mathrm{D}_{2} \mathrm{O}\right.$, Sigma-Aldrich) and then filtered using a nylon syringe filter $(0.45 \mu \mathrm{m})$ before analysis. ${ }^{1} \mathrm{H}$ NMR spectra were recorded for unsubstituted SF and GMA with 1:1 mol unsubstituted amines on SF: mol GMA.

Samples were prepared by a 3D printer for the rheological properties test (a disk with $2.5 \mathrm{~cm}$ diameter and $0.2 \mathrm{~cm}$ height), for the compression test (a disk with $1.12 \mathrm{~cm}$ diameter and $1 \mathrm{~cm}$ height), for the tensile test (a dumbbell shape sample of a concave column with $1.6 \mathrm{~cm}$ $(\mathrm{w}) \times 0.7 \mathrm{~cm}(\mathrm{~d}) \times 0.2 \mathrm{~cm}(\mathrm{~h}))$, for the swelling rate (a disk with $2.5 \mathrm{~cm}$ diameter and $0.2 \mathrm{~cm}$ height) and for the in vitro degradation study (a square sample with $1 \mathrm{~cm}$ $(\mathrm{w}) \times 1 \mathrm{~cm}(\mathrm{~d}) \times 0.2 \mathrm{~cm}(\mathrm{~h}))$. The rheological properties of $l$-Sil-MAS and $c$-Sil-MAS were measured at $25^{\circ} \mathrm{C}$ using an Anton Paar MCR 302 (Anton Paar, Zofingen, Switzerland) rheometer. The mechanical strength of the hydrogel was obtained by a universal testing machine (UTM; QM100S, QMESYS, Gunpo, Korea) that was equipped with a $10 \mathrm{kgf}$ load cell in an unconfined environment. The mechanical force was loaded at a rate of $5 \mathrm{~mm} / \mathrm{min}$ until the hydrogel broke. For the swelling property of $c$-Sil-MAS, the hydrogels were immersed in DW or PBS, and then, the swelling ratio was estimated by measuring the length of the $X$ and $Y$ axes based on the initial status immediately after printing.

In vitro degradation ${ }^{16}$ of $3 \mathrm{D}$-printed $c$-Sil-MAS was measured for 30 days using protease XIV (from Streptomyces griseus) (Pronase $\mathrm{E}^{\circledR}$, Merck, Darmstadt, Germany) with an activity of $2 \mathrm{U} / \mathrm{mL}$. Samples were immersed in $2 \mathrm{~mL}$ of PBS (pH 7.4) containing $2 \mathrm{U} / \mathrm{mL}$ protease enzyme and incubated at $37^{\circ} \mathrm{C}$. The enzyme solution was exchanged with freshly prepared solution every 3 days. The three samples were dried and weighed at predetermined time points. The degradation rate was calculated by the following formula:

$$
\text { Remaining weight } \%=\mathrm{Wt} / \mathrm{Wi} \times 100 \text {; }
$$

where $\mathrm{Wi}$ is the initial dry weight of the construct and $\mathrm{Wt}$ is the final weight after $0,3,6,9,12,15,18$, and 30 days of incubation.

\section{Adhesive mechanical tests ${ }^{17}$}

The common treatment for all adhesive tests was as follows: All tests were conducted according to the ASTM guidelines modified in terms of the size of the specimen, strain rate, or area of sample treatment. The three mechanical tests relative to adhesion of Sil-MAS $(n=3)$ were performed using a UTM that was equipped with a $3 \mathrm{kgf}$ load cell in an unconfined environment. MLB ( $n=$ 3) was used as a control. Fresh shaved rat skin from male SD rats $(180-220 \mathrm{~g})$ was prepared as a substrate tissue. This rat skin tissue was kept in PBS, and the moisture was wiped immediately before use. To attach rat skin tissue on glass slides and steel fixtures, instantaneous adhesives (LOCTITE401, Henkel, Rocky Hill, CN, USA) were used. Different quantities of sealants ( $l$-Sil-MAS and MLB) and UV treatment times for each test were used for the experiments. All tests of physical properties were performed in a room under strictly controlled conditions (80\% humidification, room temperature) to prevent drying out.

(1) In vitro lap shear test: The strength properties of sealants in lap shear by tension loading were analyzed according to the ASTM F2255-05 standard. Rat skin $(2.5 \mathrm{~cm} \times 2.5 \mathrm{~cm})$ was attached on the edge of the short side of two glass slides $(9 \mathrm{~cm} \times 2.5 \mathrm{~cm})$. Next, $300 \mu \mathrm{L}$ of each sealant was uniformly placed on the skin. Then, the other tissue-coated slide was symmetrically covered with sealants. The Sil-MAS group was irradiated with UV light $(20 \mathrm{~s})$ from every direction, and the MLB group was allowed to dry at room temperature. These assembled slides were loaded in a UTM for shear testing by tensile loading with a strain rate of $2 \mathrm{~mm} / \mathrm{min}$. The point of detachment was fixed as the maximum load. Shear strength $(\mathrm{kPa})$ was the maximum load $(\mathrm{N})$ divided by the bonding area $\left(0.5 \mathrm{~cm}^{2}\right)$. To visualize the adhesive strength of Sil-MAS, $300 \mu \mathrm{L}$ of $l$-Sil-MAS $(2.5 \mathrm{~cm} \times 2.5 \mathrm{~cm})$ was applied between a $0.9 \mathrm{~kg}$ wrench and a plastic ruler, and after UV crosslinking $(20 \mathrm{~s})$, the ruler was lifted for $2 \mathrm{~h}$.

(2) Pull-off adhesion strength: The strength properties of sealants in tension were quantified according to the ASTM F2258-05 standard. The test aims to measure the adhesive tensile strength of Sil-MAS in conjunction with the skin tissue and the force necessary to separate this bond. Two rat skin tissues $(2 \mathrm{~cm} \times 2 \mathrm{~cm})$ were attached on the steel fixtures with $2 \mathrm{~cm}$ in each dimension. Then, $300 \mu \mathrm{L}$ of each sealant was uniformly placed on the skin on the bottom side of the steel fixture. The gap between the fixtures was set at $2 \mathrm{~mm}$. The Sil-MAS group was irradiated with UV light (20 s) from every direction, and the MLB group was allowed to dry at room temperature. A transient tensile test at a constant transducer speed of $5 \mathrm{~mm} / \mathrm{min}$ was performed until complete sealant-skin substrate seal failure. The adhesion strength of sealants was determined by dividing the maximum load detected in the pull-off curve by the bonding area $\left(4 \mathrm{~cm}^{2}\right)$.

(3) In vitro wound closure strength: The wound closure strength of the sealants was tested according to the ASTM F2458-05 standard, which is specifically focused on determining the wound closure strength of medical glue. The rat skin was cut into rectangular shapes $2.5 \mathrm{~cm} \times$ $2.5 \mathrm{~cm}$ in size. The tissues were fixed onto two glass slides $(9 \mathrm{~cm} \times 2.5 \mathrm{~cm})$ with $1.5 \mathrm{~mm}$ spaces between the slides. The center of the tissue was cut using a razor blade to mimic wound injury. Then, $400 \mu \mathrm{L}$ of each sealant was uniformly applied onto the cut area $(0.5 \mathrm{~cm} \times 0.5 \mathrm{~cm})$, and 
UV light was applied to crosslink the sealant from every direction. These assembled slides were placed carefully into the UTM by tensile loading with a strain rate of $5 \mathrm{~mm} / \mathrm{min}$. The point of tearing was defined as the wound closure strength $(\mathrm{kPa})$.

\section{Ex vivo porcine aorta burst pressure test}

The burst pressure testing of Sil-MAS $(n=3)$ was conducted using a modified setup for determining vessel seal burst strength ${ }^{18}$. Briefly, fresh porcine aortas (diameter $1.8 \mathrm{~cm}$ ) from a local slaughterhouse (Hongcheon, Korea) were immersed in saline, and the experiment was carried out within $10 \mathrm{~h}$ after the aorta harvest. The aorta was cut into $15 \mathrm{~cm}$ segments, and an infusion catheter (diameter $2 \mathrm{~cm}$ ) was fitted in one opened side of the aorta. The orifice diameter of the tube was larger than that of the aorta so that the aorta covered the tube very tightly. The other opened side of the aorta was gripped by a curved mosquito clamp. The catheter was connected to a syringe pump (PILOT Anaesthesia 2, Fresenius Vial Corp., Brezins, France), which was connected to a patient monitor (MP1000NT PLUS, MEK Corp., Giheung, Korea) to verify the intraluminal pressure. Then, a small incision (3 and $9 \mathrm{~mm}$ length) was made by scalpel (\#15) in the middle of the aorta, and $200 \mu \mathrm{L}$ of $l$-Sil-MAS was placed onto the sectioned area and photocrosslinked by UV light $(20 \mathrm{~s})$.

Next, to test the subsidiary effect of Sil-MAS on suturing, the porcine aorta was cut in half using a scalpel. An aorta end-to-end anastomosis was performed using interrupted polyamide sutures (Blue Nylon, Ailee Corp., Busan, Korea). One milliliter of $l$-Sil-MAS was then applied to the anastomotic site and treated with UV light $(20 \mathrm{~s})$. The aorta sutured without sealant was used as a control. The above aortas were filled with PBS using a syringe pump through the other opened side of the aorta until the aorta was swelled by its diameter or leakage occurred at the suture site, and this point was set to zero $\mathrm{mmHg}$. Then, additional PBS was injected into the aorta using the syringe pump to increase the infusion pressure. The aorta was swollen under continuous PBS injection, and the burst pressure $(\mathrm{kPa})$ of Sil-MAS was recorded at the point of material burst, detachment or tissue burst.

\section{Animal study}

\section{In vivo study using a rat model}

Common treatment for all rat tests: The animal studies were carried out in accordance with guidelines and approval of the Institutional Animal Care and Use Committee (IACUC) of Hallym University in Korea (\# Hallym 2018-11). Healthy male Sprague-Dawley (SD) rats each weighing 180-220 g were used (Samtako, Osan, Korea). After being raised under experimental conditions for 1 week, the rats were randomly divided into $1-3$ groups depending on the analysis. Anesthesia was induced with $3 \%$ isoflurane in a 75:25 mixture of nitrous oxide and oxygen in a standard induction chamber with a gas vaporizer (Harvard Apparatus, South Natick, MA, USA) and maintained at $2 \%$, administered via a small rodent respirator. Each group was tested in triplicate. For animal sacrifices, the rats were euthanized with a lethal dose of thiopental urethane.

(1) Wound closure animal study: To evaluate the adhesive property of Sil-MAS, the backs of rats $(n=3)$ were shaved. After disinfection, skin incisions $1.5 \mathrm{~cm}$ long and partial-skin thickness deep were made on the backs of rats by a surgical blade (\#10). $l$-Sil-MAS $(200 \mu \mathrm{L})$ was quickly applied to the wound, with fingers used to slightly hold both ends of the incision, and then crosslinked with UV light for $10 \mathrm{~s}$. Next, sealing by Sil-MAS was confirmed by gently pulling the sealed site in the opposite direction of incision with two fingers.

(2) Hemostasis and wound healing in an in vivo partialthickness skin wound model: The rats were divided into three groups: the Sil-MAS group $(n=3)$, the Avitene ${ }^{\mathrm{TM}}$ group $(n=3)$ and the gauze group $(n=3)$. Commercial artificial dermis Avitene ${ }^{\mathrm{TM}}$ and standard gauze as controls were cut into $1 \mathrm{~cm} \times 1 \mathrm{~cm}$ square shapes. After shaving and disinfection of the backs of rats, in the rostral-to caudal direction, three $1 \mathrm{~cm} \mathrm{(w)} \times 1 \mathrm{~cm}(\mathrm{~d}) \times 0.3 \mathrm{~cm}(\mathrm{~h})$ partial-thickness skin defects per animal were cut out with a homemade tool. Through this procedure, the epidermis and partial dermis were removed. One of the three wounds was covered with Avitene ${ }^{\mathrm{TM}}$, and another was covered with gauze. The other wound was sequentially treated with $l$-Sil-MAS $(200 \mu \mathrm{L})$ and UV light $(25 \mathrm{~s})$. To secure the treatment, Surgifix ${ }^{\circledR}$ (BSN medical, Victoria, Australia) was wrapped around the animal from below the forelimbs to above the hindlimbs. The wound site was photographed on the 0 , 3rd, 7th, 14th, and 21st days, and the remaining wound area was measured and converted to a percentage based on the initial wound area. After photography, rats were sacrificed, and full-thickness skin tissue was harvested at the initial wound size and divided into two equal portions for western blotting and histology analysis.

(3) Femoral artery hemorrhage: The hair of rats $(n=3)$ was removed over the right inguinal region. After disinfection, the skin and subcutaneous tissues were cut to expose the femoral vein and artery. Bleeding was produced by incision of the femoral artery and vein using a sterilized scalpel. After free bleeding was observed for $5 \mathrm{~s}$, the damaged area was pressed gently with gauze for $3 \mathrm{~s}$. The gauze was removed, and immediately, $l$-Sil-MAS $(50 \mu \mathrm{L})$ and UV light $(20 \mathrm{~s})$ were sequentially applied to the wound.

(4) Subcutaneous implantation: For in vivo degradation tests and biocompatibility tests, samples $(1.5 \mathrm{~cm}(\mathrm{w}) \times 1.5 \mathrm{~cm}$ 
(d) $\times 0.2 \mathrm{~cm}(\mathrm{~h}))$ were printed out by a 3D DLP printer. The backs of rats were shaved and disinfected. Two incisions $1 \mathrm{~cm}$ long were created on the rostral side and caudal side on the dorsal midline of the backs of the rats. The separated subcutaneous pockets were made using Metzenbaum scissors. Printed $c$-Sil-MAS samples were placed in the subcutaneous pockets. At 2, 4, 10, and 16 weeks after implantation, the rats were euthanized, and the implanted $c$ Sil-MAS samples were harvested with the surrounding tissue. Explanted samples were washed with DW. For the degradation test $(n=3)$, excess tissue surrounding the samples was carefully removed. In vivo degradation was measured based on the sample size before and after implantation using a stereomicroscope (SMZ25, Nikon Co., Tokyo, Japan).

(5) Liver hemorrhage: For liver hemostasis surgery, rats were divided into two groups: the Sil-MAS group and the fibrin glue group. An abdominal midline incision was made after local disinfection. The liver was exposed and separated from the surrounding tissues by a retractor. Two of the three lobes of the liver were scraped out $(0.5 \mathrm{~cm}$ deep and $0.5 \mathrm{~cm}$ diameter) using tissue forceps and a scalpel after punching with a tissue punch (diameter $0.5 \mathrm{~cm}$ ). After free bleeding for $5 \mathrm{~s}$, the damaged area was pressed gently with gauze for $3 \mathrm{~s}$. Then, $l$-Sil-MAS $(200 \mu \mathrm{L})$ and UV light $(20 \mathrm{~s})$ were sequentially applied to the wound immediately after removal of gauze. The fibrin sealant was prepared and applied to the wound according to the manufacturer's instructions. At 1, 2, 4, or 8 weeks after surgery, the rats were euthanized, and the sealanttreated region, including peri-implant tissue, was resected from the rats for further histological analysis.

\section{Laparoscopic surgery in a rabbit laceration model of liver and stomach serosa}

Laparoscopic surgery was performed in a female New Zealand white rabbits weighing $2.8 \mathrm{~kg}$. Anesthesia was induced with intramuscular ketamine $(250 \mathrm{mg} / 5 \mathrm{~mL}$, Huons, Seongnam, Korea) and Rompun injection (23.32 mg/mL, Bayer Korea, Seoul, Korea) and maintained with inhalational sevoflurane (2\%) and oxygen mixed with room air $(1.5 \mathrm{~L} / \mathrm{min})$ using a ventilator (Veterinary anesthesia ventilator, Solar Medical Tech, Taipei, Taiwan). The study was approved by the IACUC of Hallym University in Korea. A rabbit was secured to the table in the supine position. The $\mathrm{CO}_{2}$ insufflator and camera system with a $4 \mathrm{~mm} 0^{\circ}$ endoscope (Karl Storz, Tuttlingen, Germany) was combined with the complete laparoscopic unit (MGB, Berlin, Germany). Three $5 \mathrm{~mm}$ laparoscopic trocars (Laport, Sejong Medical, Paju, Korea) were introduced into the abdominal cavity, and the insufflation pressure was established as $8-12 \mathrm{mmHg} \mathrm{CO}_{2}$ gas. The instruments used were endoscopic scissors, forceps, a suction-irrigation probe, a homemade endoscopic sealant device with fiber optics and a microsyringe pump. Multiple superficial or deep liver laceration wounds were made by endoscopic scissors. Bleeding liver laceration lesions were briefly pressed with gauze, and $l$-Sil-MAS $(1 \mathrm{~mL})$ and UV light $(20 \mathrm{~s})$ were applied by a homemade endoscopic sealant device. Rebleeding or leakage of blood at the lesions was checked using an endoscopic camera and forceps. In the same way, through laparoscopic operation, laceration of stomach serosa, Sil-MAS treatment, and checking the result were performed.

\section{Western blotting}

Twenty milligrams of frozen tissue (frozen with liquid nitrogen) was mixed with $1 \mathrm{~mL}$ of ice-cold RIPA buffer (with protease, dephosphorylase inhibitor cocktail) and homogenized using a hand homogenizer (Super Fast Prep- $2^{\mathrm{TM}}$, MP Biomedicals, Santa Ana, CA, USA). Then, the mixture was incubated at $4{ }^{\circ} \mathrm{C}$ for $2 \mathrm{~h}$ with agitation, and the supernatants were collected by centrifugation for $20 \mathrm{~min}$. The protein concentration in the supernatants was measured using a Bradford assay (Bio-Rad, Hercules, CA, USA). The same amount of denatured protein sample was then loaded and separated on an SDS-polyacrylamide gel and transferred to a PVDF membrane (Millipore, Billerica, MA, USA). Nonspecific binding was blocked using $10 \%$ skim milk for $2 \mathrm{~h}$ at room temperature. Then, the membrane was incubated with primary antibodies (1:1000) overnight at $4{ }^{\circ} \mathrm{C}$. Primary antibodies for vimentin, fibronectin, VEGF, and cyclin D1 were purchased from Abcam (Cambridge, UK). $\beta$-Actin antibody was supplied by Sigma-Aldrich and used at 1:5000. Afterward, the membrane was incubated at room temperature for $1 \mathrm{~h}$ with species-matched secondary antibodies (goat anti-rabbit and goat anti-mouse IgG) (Enzo Life Sciences, Farmingdale, NY, USA). Finally, bands were detected by a chemiluminescence system (Fusion FX, Vilber Lourmat). Experiments were conducted using tissue samples from three different rats.

\section{Histology}

The harvested samples were fixed with $4 \%$ paraformaldehyde for $24 \mathrm{~h}$, dehydrated in graded alcohols and xylene and then embedded in paraffin. Tissue samples five microns thick were prepared and stained with hematoxylin and eosin (HE) to analyze the re-epithelialization and inflammatory response and Masson's trichrome (MT) to detect collagen production. Furthermore, to check tissue compatibility, samples harvested from liver and subcutaneous tissue at each time point were immunostained with anti-CD68 (Abcam, Cambridge, MA, USA) and antiCD31 (Novus Biologicals, Centennial, CO, USA) primary antibodies and then with DyLight ${ }^{\circledR}$ 550-conjugated (Bethyl Laboratories, Montgomery, TX, USA) and FITCconjugated secondary antibodies, respectively. The slides 
were mounted by Vectashield antifade mounting medium with DAPI (Vector Laboratories, Burlingame, CA, USA). Stained sections were analyzed under a fluorescence microscope (Eclipse 80i, Nikon Co.).

\section{Cytocompatibility assay and cell migration assay}

To test the cytotoxicity of Sil-MAS depending on the UV treatment time and wound healing effect of Sil-MAS in vitro, the elution test method was applied. Two milliliters of $l$-Sil-MAS was prepared in 6-well plates, and UV treatment was administered for 10,20 , and $30 \mathrm{~s}$. Then, $10 \mathrm{~mL}$ of a growth medium consisting of Dulbecco's modified Eagle medium (DMEM) with 10\% (v/v) fetal bovine serum and $1 \%(\mathrm{v} / \mathrm{v})$ penicillin-streptomycin was added and incubated in a $5 \% \mathrm{CO}_{2}$ incubator at $37^{\circ} \mathrm{C}$ for $72 \mathrm{~h}$. This medium (Sil-MAS conditioned medium) was extracted and sterilely filtered with a cellulose acetate syringe filter $(0.2 \mu \mathrm{m})$ to preserve all living cells. NIH/3T3 mouse fibroblast cells (Koram Biotech, ATCC distributor, Seoul, Korea) were cultured in the above growth medium. All cultures were maintained in a $5 \% \mathrm{CO}_{2}$ incubator at $37^{\circ} \mathrm{C}$ with the medium changed every three days. Cells were detached using trypsin-EDTA $0.25 \%$, and 5,000 cells/well were plated in 24-well plates. After stabilization of cell attachment, the culture medium was replaced with $200 \mu \mathrm{L}$ of the filtered Sil-MAS conditioned medium and cultured for 3 days without medium changes. The cell viability depending on culture time was examined by CCK-8 assay (Dojindo Molecular Technology, Rockville, MD, USA) according to the manufacturer's protocol. As a positive control group, $200 \mu \mathrm{L}$ of SF solution prepared at a concentration of $25 \%(\mathrm{w} / \mathrm{v})$ in the growth medium was added to cells.

For the wound healing assay in vitro, NIH/3T3 cells were cultured until the confluence reached approximately $80-90 \%$. A scratch was created using $200 \mu \mathrm{L}$ micropipette tips. Immediately after the scratch, floating cells were removed by PBS washing, and $200 \mu \mathrm{L}$ of Sil-MAS conditioned medium and growth medium (control) was added to each well. While cells were being cultured, images were captured at $3 \mathrm{~h}$ intervals for $15 \mathrm{~h}$ with a digital camera (EOS 100D, Canon, Tokyo, Japan) through an optical microscope (Eclipse 80i, Nikon Co.). The rate of migration was quantified through the total distance that the NIH3T3 cells moved toward the center of the wound from the edge of the wound within $15 \mathrm{~h}$.

\section{Statistical analysis}

Samples for each group were assessed in triplicate to analyze the statistical data. The data are presented as the mean value \pm standard deviation (s.d.) $\quad(" p<0.05$, * $p<$ $0.01,{ }^{* * * * *} p<0.001$, and $\left.{ }^{* * * * *} p<0.0001\right)$. Curve fitting was performed using Prism, version 6.0 software (GraphPad, San Diego, CA, USA), and one-way ANOVA followed by the Tukey multiple comparisons test was used to assess significant differences among multiple groups.

\section{Results and discussion \\ Fabrication of Sil-MAS}

Figure 1a presents a schematic diagram illustrating the synthetic process of the prepolymer for Sil-MAS, which relies on the reaction of GMA with SF, as well as the chemical structure of Sil-MAS according to the conditions. In the synthesis, the photoreactive vinyl groups of GMA responsible for UV crosslinking were mainly conjugated to the primary amine groups of SF after epoxide ring opening, resulting in secondary or tertiary amines with hydroxyl groups. The modification of SF by GMA was confirmed through NMR spectroscopy (Fig. S1). Creation of $l$-Sil-MAS was completed by the addition of LAP as a photoinitiator to the Sil-MA solution immediately before use. Finally, $l$-Sil-MAS was photocrosslinked (c-Sil-MAS) on the target by UV irradiation at $365 \mathrm{~nm}$. LAP produces free radicals following light treatment, causing $l$-Sil-MAS to become crosslinked via free radical vinyl polymerization. In this study, Sil-MAS was tested for various external and internal uses; Fig. 1b depicts a schematic drawing of the applications of Sil-MAS such as the treatment of damaged skin, liver, blood vessels, and stomach.

\section{Adhesion properties of Sil-MAS}

The detailed physical properties of Sil-MAS, such as the mechanical, rheological and degradable properties, are presented in the Supporting Information (Table S1, Fig. S2-S4). $l$-Sil-MAS shows a suitable viscosity for handling, a proper mechanical strength for internal organs, a low swelling ratio to avoid affecting surrounding organs, and a slow degradation rate (slower than that of commercial sealants ${ }^{2}$ ) suitable for long-term sealing effects on targets.

Adhesion tests to measure the lap shear strength, tensile adhesion strength and wound closure strength of sealants were performed (Fig. 2). Medifoam ${ }^{\circledR}$ Liquid bandage (MLB, Mundipharma), which is a commercially available active sealant in Korea, was used as a control. A lap shear test was applied based on the ASTM F2255-05 standard to determine the shear strength of sealants (Fig. 2a). In addition to performing the skin test, we carried out the test on steel without tissue because the biological membranes themselves have inconsistent surface properties and vary by sample. On both surfaces, Sil-MAS showed higher shear strain and shear stress at break than MLB. Reflecting the high resistance to shear, the adhesive strength of Sil-MAS was strong enough to lift and hold a wrench $(0.9 \mathrm{~kg})$ for prolonged time periods (Fig. $2 \mathrm{~b}$, Video S1). To test the strength properties of sealants in tension, a pull-off adhesion test was performed using modified ASTM F2258-05 (Fig. 2c). Similar to the results of the 


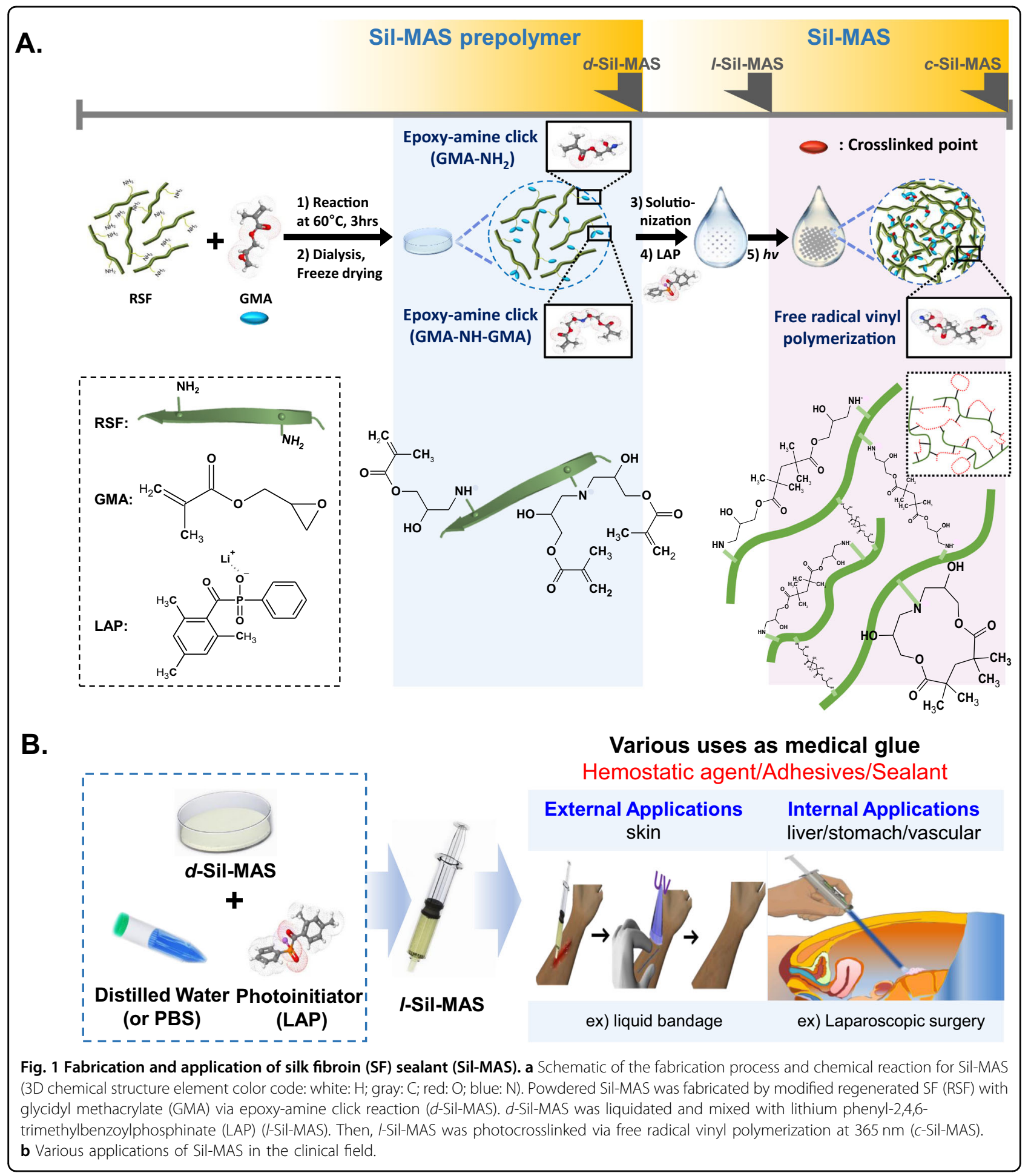

lap-shear test, Sil-MAS produced higher tensile strength and higher elongation at break than MLB. The wound closure strength of sealants was assessed using modified ASTM F2458-05 (Fig. 2d). Both the highest adhesive strength and elongation of Sil-MAS were shown to be higher than those of MLB. In addition, Sil-MAS was superior to MLB at both the breaking stress and breaking strain points, which means that Sil-MAS has higher ductile properties, lower brittleness, and greater toughness. To test the effectiveness of Sil-MAS for in vivo sealing without any surgical methods, a rat model of skin incision was used (Fig. 2e, Video S2). Skin incisions $1.5 \mathrm{~cm}$ 
A.

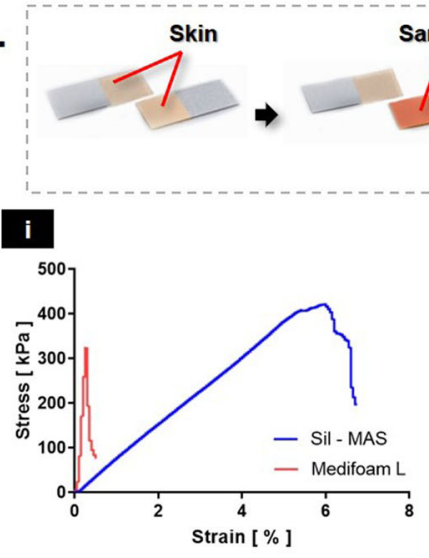

c.
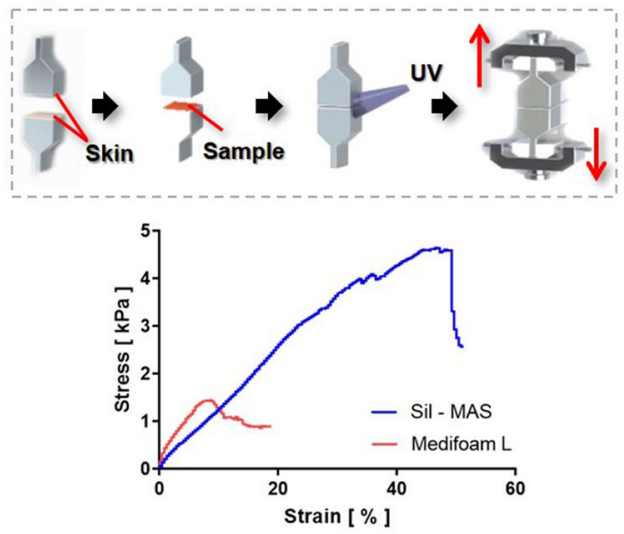

E.

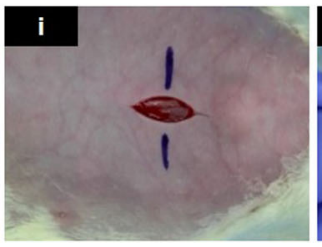

\section{if}

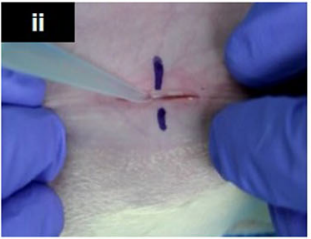

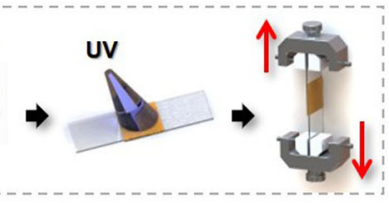

ii

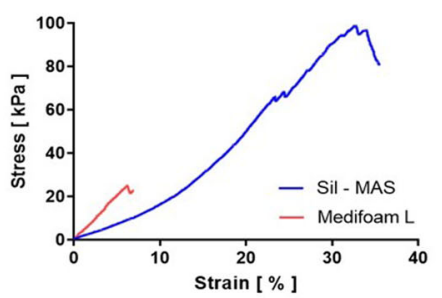

D.
B.
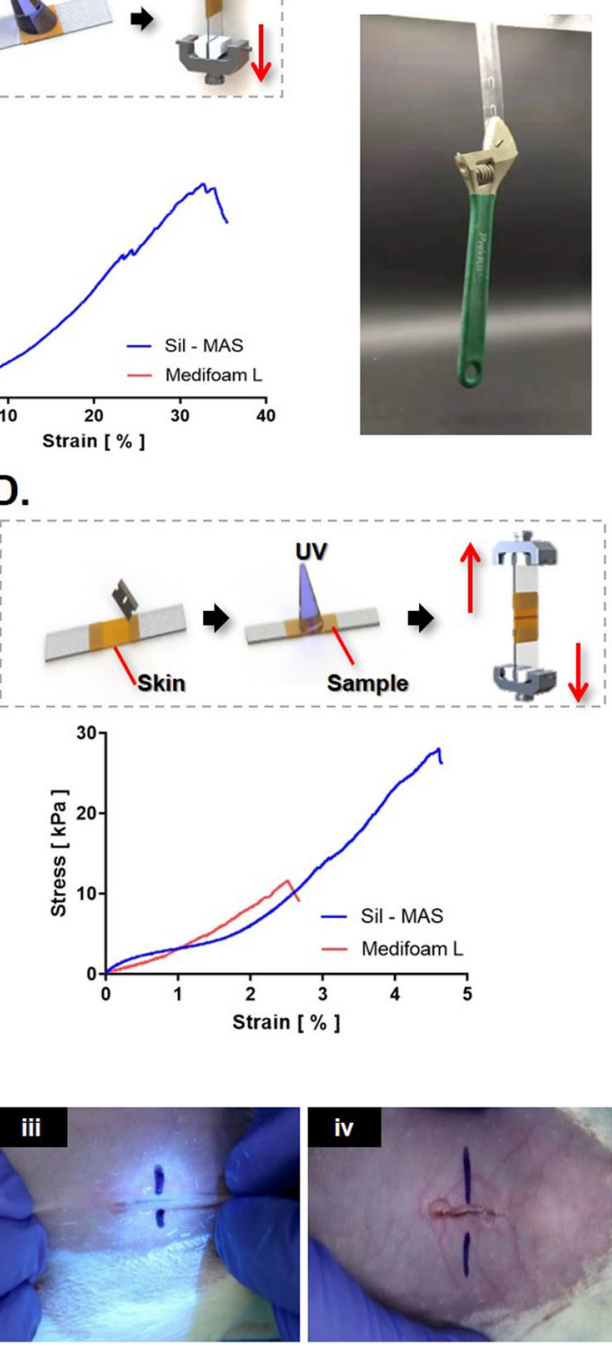

\section{iv}

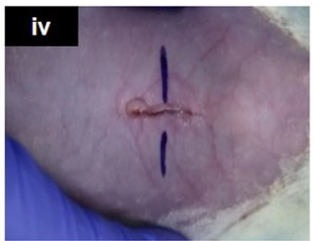

Fig. 2 Adhesion properties of Sil-MAS ( $\boldsymbol{n}=\mathbf{3})$. Medifoam " Liquid bandage (MLB, $n=3)$ was used as a control. a Schematic of the measurement procedure for the strength property relative to lap shear and its representative stress/strain curve. Comparison of Sil-MAS and MLB on (i) steel and (ii) rat skin, showing similar trends. b Visualization of Sil-MAS adhesive strength. Sil-MAS was applied and cured between a $0.9 \mathrm{~kg}$ wrench and a plastic ruler, and the ruler was lifted. c Schematic of the measurement procedure for pull-off strength and its representative stress/strain curve. $\mathbf{d}$ Schematic of the measurement procedure for wound closure strength and its representative load/extension graph. e Photographs of the in vivo rat wound closure test. (i) Skin incisions $1.5 \mathrm{~cm}$ long and partial-skin thickness deep were made on the backs of the rats by a surgical blade. (ii) The skin incisions were held together with two fingers. (iii) The wound was quickly treated with $200 \mu \mathrm{L}$ of I-Sil-MAS. (iv) The I-Sil-MAS was crosslinked by UV light for $10 \mathrm{~s}$. Then, the sealed site was gently pulled apart by two fingers to check adhesion and sealing by Sil-MAS.

in length and partial skin thickness in depth were successfully sealed via crosslinking of $l$-Sil-MAS. The sealed site was not torn when pulled gently with fingers.

\section{Biocompatibility of Sil-MAS in vitro and in vivo}

The biocompatibility of Sil-MAS was tested both in vitro and in vivo. Sil-MAS showed high cell viability regardless of UV treatment time (10-30 s) on Sil-MAS (Fig. 3a). The longer the sealant was crosslinked by UV light, the higher the cell viability and the better the proliferation. However, there was no significant difference among the groups, and even the result for the Sil-MAS group treated by UV for only $10 \mathrm{~s}$ was similar to that of treated pure SF. The tissue response following subcutaneous implantation of $c$-Sil-MAS in rats was histologically examined through HE staining and immunohistochemistry (IHC) for CD68 over the subsequent 16 weeks (Fig. $3 \mathrm{~b}$ ). An increase in the number of vascular structures and the formation of collagenous tissue accompanied by a decrease in the number of 
A.

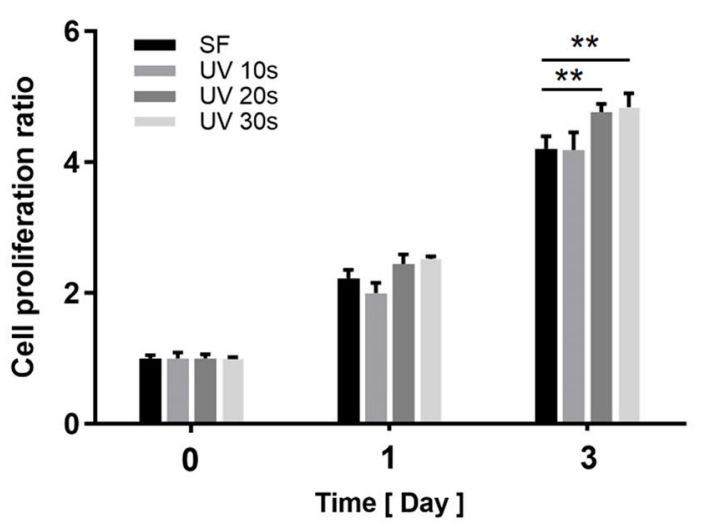

B.

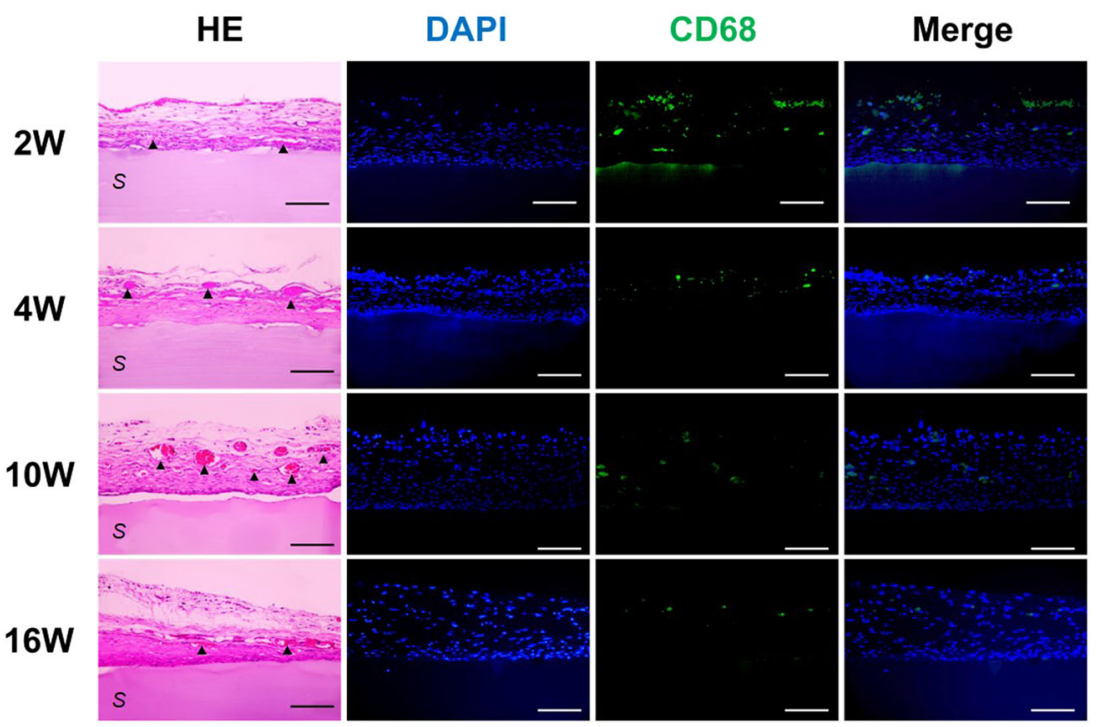

Fig. 3 Biocompatibility of Sil-MAS in vitro and in vivo. a In vitro cytotoxicity of Sil-MAS depending on UV crosslinking time (10, 20, and 30 s). Pure SF solution was used as a positive control group. Data are reported as the means \pm SD $\left({ }^{* *} p<0.01 ; n=3\right)$. b In vivo biocompatibility test using a rat subcutaneous model. Histological evaluations of tissue neighboring the implanted c-Sil-MAS stained with HE and immunostaining to visualize macrophages (CD68). HE staining revealed that the numbers of vascular structures (black arrowheads) and fibroblasts in the newly formed tissue around sealant were increased up to 10 weeks and decreased at 16 weeks. The number of macrophages in the collagenous tissue decreased over time. See Supporting Information for more detailed analysis.

macrophages with time were observed, which reflects the biocompatibility of Sil-MAS.

\section{Hemostasis and wound healing effect of Sil-MAS in an in vivo partial-thickness skin wound model}

We evaluated the hemostasis and healing properties of Sil-MAS in an acute partial-thickness wound rat model (Fig. S5, Video S3). In the partial-thickness wound, bleeding substantially stopped following application of $l$ Sil-MAS and completely stopped following UV curing. The advantages of the application of Sil-MAS were not limited to hemostasis following abrasion. It was hypothesized that wound healing can progress naturally without removal of the Sil-MAS covering because $c$-SilMAS has high viscoelasticity and SF possesses good biocompatibility and an intrinsic wound healing effect ${ }^{3}$. Photographic records were generated to analyze the macroscopic wound closure at days $0,3,7,14$, and 21 after treatment with Sil-MAS, Avitene ${ }^{\mathrm{TM}}$ (control group 1) and gauze (control group 2) on rat partial-thickness wounds (Fig. S6). The Sil-MAS covering on the wound peeled off as the skin was repaired. Moreover, it was confirmed that the wound site in the Sil-MAS treatment group had a smooth and hairy surface without wrinkles from the third day. This result proves that Sil-MAS administered on the wound does not need to be replaced because it adheres well to the injured tissue without sticking, absorbs exudate, promotes wound healing and peels off spontaneously as the wound heals ${ }^{3}$. Quantitatively, the wound area in the Sil-MAS group decreased the 


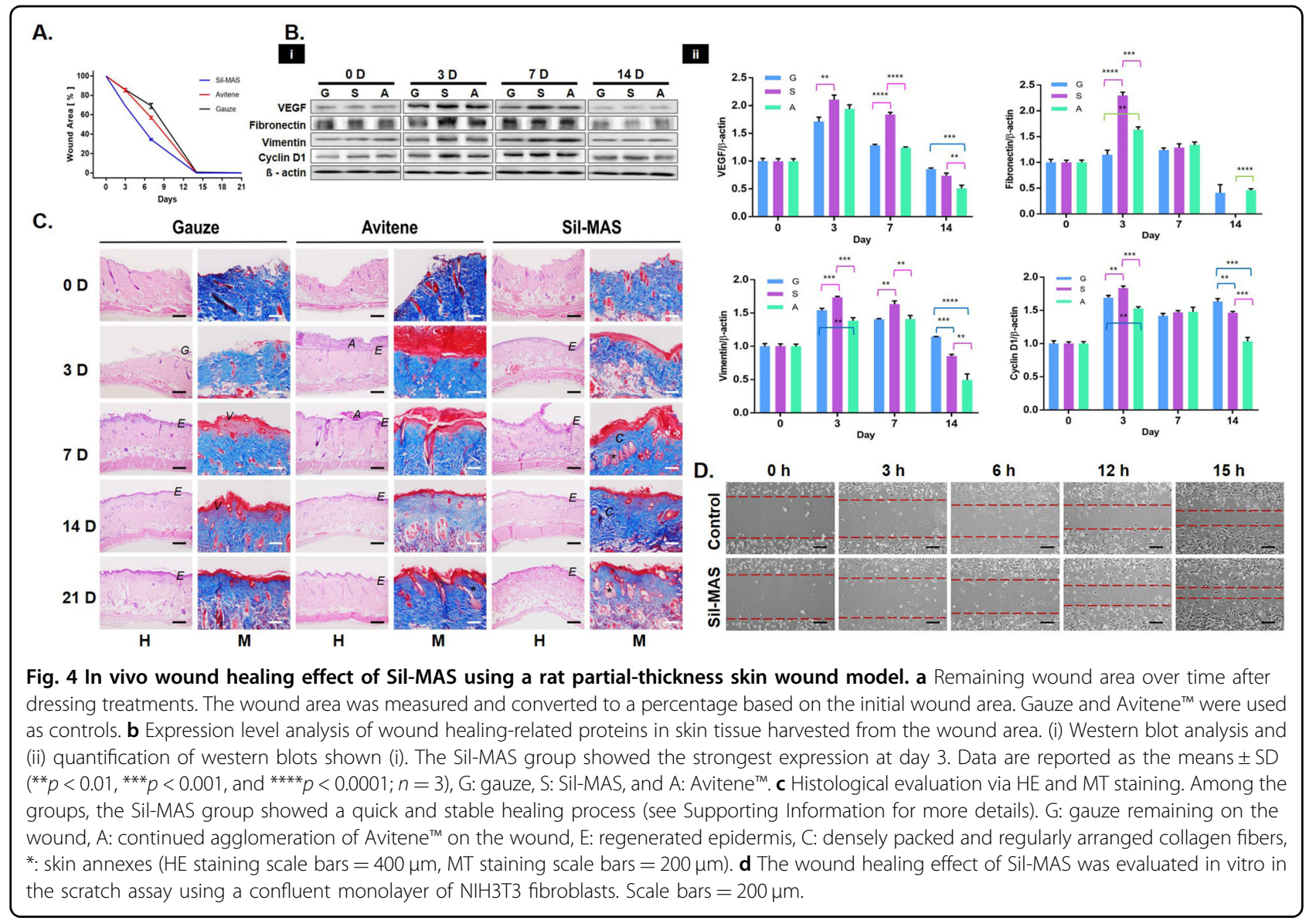

fastest among the groups (Fig. 4a). To further confirm whether Sil-MAS promotes skin regeneration, the protein expression levels of wound healing-related factors were assessed using western blot analysis (Fig. 4Bi). Vascular endothelial growth factor (VEGF) promotes wound healing by several mechanisms such as collagen deposition, angiogenesis and epithelization ${ }^{19}$. Fibronectin is an adhesive molecule that contributes to wound healing, including extracellular matrix formation and reepithelialization $^{20}$. Vimentin has been shown to be involved in tissue repair and regeneration including cell migration, proliferation, differentiation, angiogenesis, extracellular matrix remodeling and immune responses ${ }^{21}$. Finally, cycle regulatory protein (cyclin D1) is associated with cell proliferation and wound healing ${ }^{22}$. Western blot analysis showed that in all groups, there were increased levels of VEGF, fibronectin, vimentin, and cyclin D1 in the wound tissue at days 3 and 7 compared with immediately after wounding. Of note, in the Sil-MAS group at day 3, all proteins were found to be expressed the most among all of the groups tested, and the expression levels also reached their peaks during the test period (Fig. 4Bii). This result indicated that Sil-MAS promoted the wound healing process through upregulation of these proteins, which is in agreement with our previous studies that SF solution increased the cell migration of primary human dermal fibroblasts and induced the expression of the same proteins in vivo ${ }^{22}$. These studies demonstrate that the major component SF within Sil-MAS could affect the wound healing process.

We assessed the histological features of wound healing in rats, including the re-epithelialization, inflammatory response, deposition of collagen and regeneration of adnexal structures, through HE and MT staining (Fig. 4c). Briefly, overall, Avitene ${ }^{\mathrm{TM}}$ and Sil-MAS showed quick and stable healing based on rapid regrowth of epithelium, well-arranged and dense collagen, large numbers of fibroblasts, and a rich reconstitution of adnexal structures. However, the Avitene ${ }^{\mathrm{TM}}$ group retained a few agglomerations of Avitene ${ }^{\mathrm{TM}}$ until 7 days posttreatment. The gauze group showed the slowest healing among groups, exhibiting late, irregular and immature re-epithelization, fewer fibroblasts, less vascular structure and more loosely packed collagen. The wound healing effect of Sil-MAS was doubly evaluated in vitro using a wound scratch assay (Fig. 4d). The scratched gap of NIH3T3 cells in the SilMAS group narrowed more rapidly than that in the medium-treated group. In light of these results, Sil-MAS 
could serve as an improved occlusive dressing for healing following skin abrasion or split-thickness donor site healing.

\section{Vascular closure effect and hemostatic effect of Sil-MAS on wet surfaces}

Vascular injury is one of the main causes of death and disability, and hemostatic control from damaged arteries is essential for successful surgery. To evaluate the efficacy of Sil-MAS on vascular hemostasis and vascular sealing, burst pressure testing ex vivo was performed (Fig. 5a). The burst pressure after Sil-MAS treatment was within the range of physiological systolic arterial pressure in humans (90-140 mmHg) (Fig. 5b, Video S4). We further confirmed a potential burst pressure improvement by SilMAS after aorta end-to-end anastomosis made with interrupted sutures (Fig. 5c). The joining strength provided by Sil-MAS was superior (1.5 times) to that provided by nylon suture only. However, the use of Sil-MAS would be limited to repairing veins or capillaries (3-30 $\mathrm{mmHg}$ ) or combined with direct arterial suture repair because arterial blood pressure can exceed $200 \mathrm{mmHg}$ during vascular surgery. Figure 5d shows snapshots from the rat femoral artery hemorrhage experiment. In the rat vascular incision model, the bleeding completely stopped after treatment with SilMAS (Video S5). Finally, Sil-MAS was applied to a rat liver using a parenchymal injury model as a potential clinical application to determine its effect on hemostasis, wound healing and biocompatibility. The bleeding was easily stopped by sequential treatment with $l$-Sil-MAS and UV light (Fig. 5e, Video S6). Fibrin glue also stopped the bleeding after thrombin crosslinking; however, it required a longer polymerization time $(<60 \mathrm{~s})$ than Sil-MAS $(<30 \mathrm{~s})$. This additional time led to dispersal of the fibrin glue and increased the risk of fibrin sealant liquid pooling $^{2}$. The fibrin glue almost disappeared completely at 4weeks posttreatment (Fig. 5f). On the other hand, the SilMAS group showed a glistening appearance of $c$-Sil-MAS at 4 weeks, which progressively decreased over time.

\section{Wound healing effect and inflammatory response of Sil- MAS in a liver punch biopsy model}

To identify the wound healing and in vivo response surrounding the implants, we harvested wound beds from the rat livers at $1,2,4$, and 8 weeks after surgery and performed histological examination. Tissue remodeling via wound healing and material degradation were analyzed following HE staining (Fig. 6a). In both the Sil-MAS and fibrin glue groups, stable collagenous tissue and normal liver tissue were found with prolonged time. Vascular structures in the newly formed tissue and cell infiltration into the materials and material absorption were found to be more abundant and to occur earlier in the fibrin glue group than in the Sil-MAS group over a period of 8 weeks.

Since endothelial cells and macrophages play a critical role in the inflammatory and healing process ${ }^{23}$, the presence of CD31 and CD68 was verified by double immunofluorescent staining (Fig. 6b). Both CD31 and CD68 were detected in greater numbers and earlier in the fibrin glue group than in the Sil-MAS group, reflecting more rapid material absorption during the test. The level of endothelial cells and macrophages in the Sil-MAS group was consistent with a mild inflammatory response. These long-term histological findings indicate that Sil-MAS forms natural and stable collagen and does not induce a chronic inflammatory response in vivo except for a mild inflammatory response consistent with normal healing. Such slight differences between fibrin glue and Sil-MAS might result from their different degradation rates.

\section{Laparoscopic surgery using a homemade laparoscopic Sil- MAS device in a rabbit laceration model of liver and stomach serosa}

Endoscopic or laparoscopic surgery is the preferred surgical approach for patients due to less postoperative pain, better cosmetic results, a shorter duration of hospital stay, and earlier return to work than those of other approaches $^{24}$. Even though this surgical procedure is currently frequently performed, it has numerous complications such as bleeding from the operation $\operatorname{site}^{25}$. In this evaluation, we present a proof of concept that Sil-MAS could be a suitable photocuring laparoscopic medical glue via laparoscopic surgery. Therefore, we fabricated an endoscopic device specially designed for laparoscopic surgery, which consists of $l$-Sil-MAS and a light source (Fig. 7a). This device is designed for easy manipulation and rapid gelation with minimal blood loss inside the body. Last, we applied this system to a rabbit laceration model of liver and stomach serosa through laparoscopic surgery. l-Sil-MAS was easily administered via the laparoscopic surgery device and successfully achieved gelation, adhesion, and hemocoagulation on wet liver tissue via UV treatment (Fig. 7b, Video S7). Likewise, SilMAS performed adequately in the laceration model of stomach serosa, which affords the possibility of application to all intestinal lesions through fast and strong adhesive effects (Fig. 7c, Video S8). These results proved that Sil-MAS represents an ideal surgical glue and a viable option to improve the efficiency of laparoscopic operation. In addition, the developed laparoscopic surgery device of an all-in-one sealant/light source would be invaluable to deliver photocurable sealants including SilMAS.

As noted above, Sil-MAS was applied well to tissues both externally (skin) and internally (vascular structures, liver, and stomach) with rapid photocrosslinking 
A.

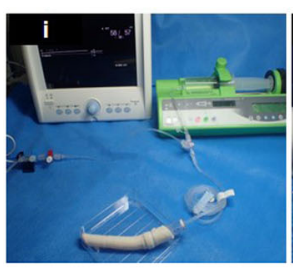

$3 \mathrm{~mm}$

B.

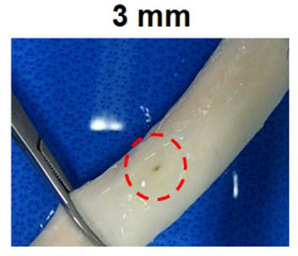

Nylon

C.

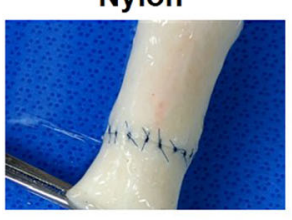

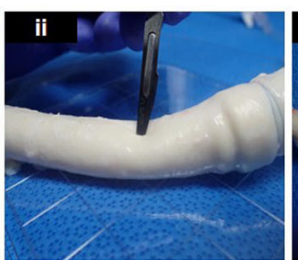

$9 \mathrm{~mm}$

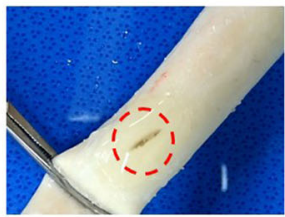

Nylon + Sil-MAS

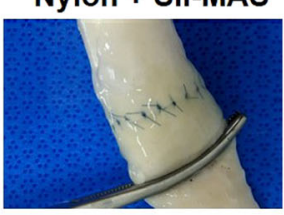

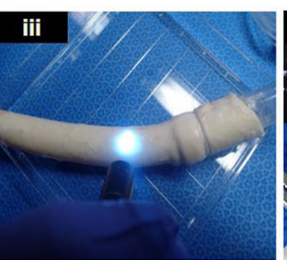
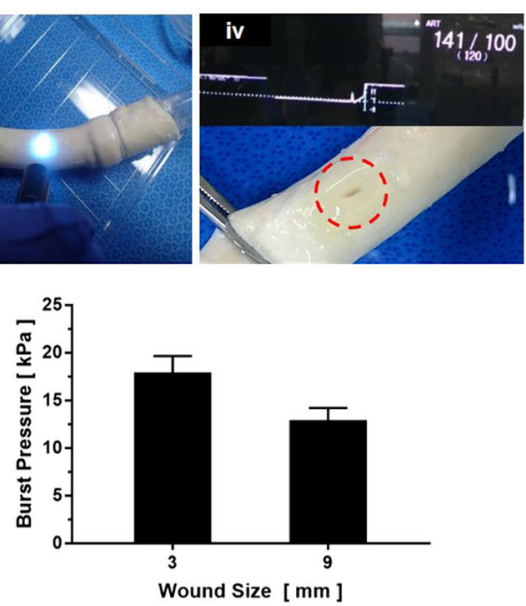

**

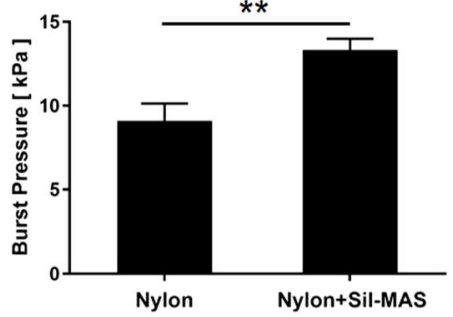

D.
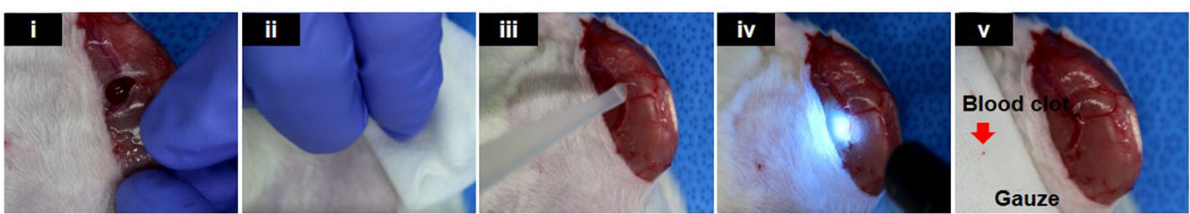

E.
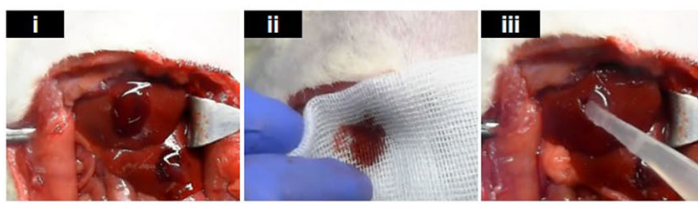

iv
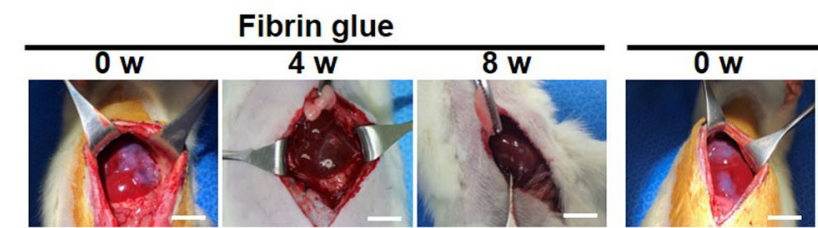

Sil-MAS

\section{F.}

Fig. 5 Vascular closure effect and hemostatic effect of Sil-MAS on wet surfaces using small and large animal models. a Illustration of the ex vivo porcine aorta burst pressure test procedure for Sil-MAS. (i) The setup for the aorta burst pressure test, (ii) incision in the middle of the aorta segment, (iii) application of I-Sil-MAS over the incision site and UV curing. $\mathbf{b}$ Porcine aorta burst pressures on the partial incision model. c Porcine aorta burst pressures on the aorta end-to-end anastomosis model. Data are reported as the means \pm SD $\left({ }^{* *} p<0.01 ; n=3\right)$. $\mathbf{d}$ In vivo vascular closure test with Sil-MAS using a rat incision model of femoral artery (i) incision and bleeding of femoral artery, (ii) slight hemostasis with gauze, (iii) application of I-Sil-MAS over the incision site, (iv) UV curing, and (v) confirmation of hemostasis. e Photographs of sealants applied in an in vivo rat liver parenchymal injury model. (i) Bleeding from the injury site arose after the creation of a circular tissue extraction with a $0.5 \mathrm{~cm}$ long and $0.5 \mathrm{~cm}$ deep injury on the surface of the rat liver, (ii) dabbing the wound with gauze for light hemostat, (iii) application of Sil-MAS (or fibrin glue) to the resected site, (iv) crosslinking by UV irradiation (for Sil-MAS) and thrombin (for fibrin glue), and (v) confirmation of bleeding cessation. $\mathbf{f}$ Photographs of sealant (Sil-MAS and fibrin glue)-treated sites in the liver parenchymal injury model at 0, 4, and 8-weeks posttreatment.

(within $30 \mathrm{~s}$ ). The rapid gelation created a physical barrier to protect the wound site, contributed to successful hemostasis, and produced a strong bridge between torn tissues, even for wet tissue such as the liver. We believe that this material's strong adhesive properties result from a combination of mechanical interlocking ${ }^{17}$ by 


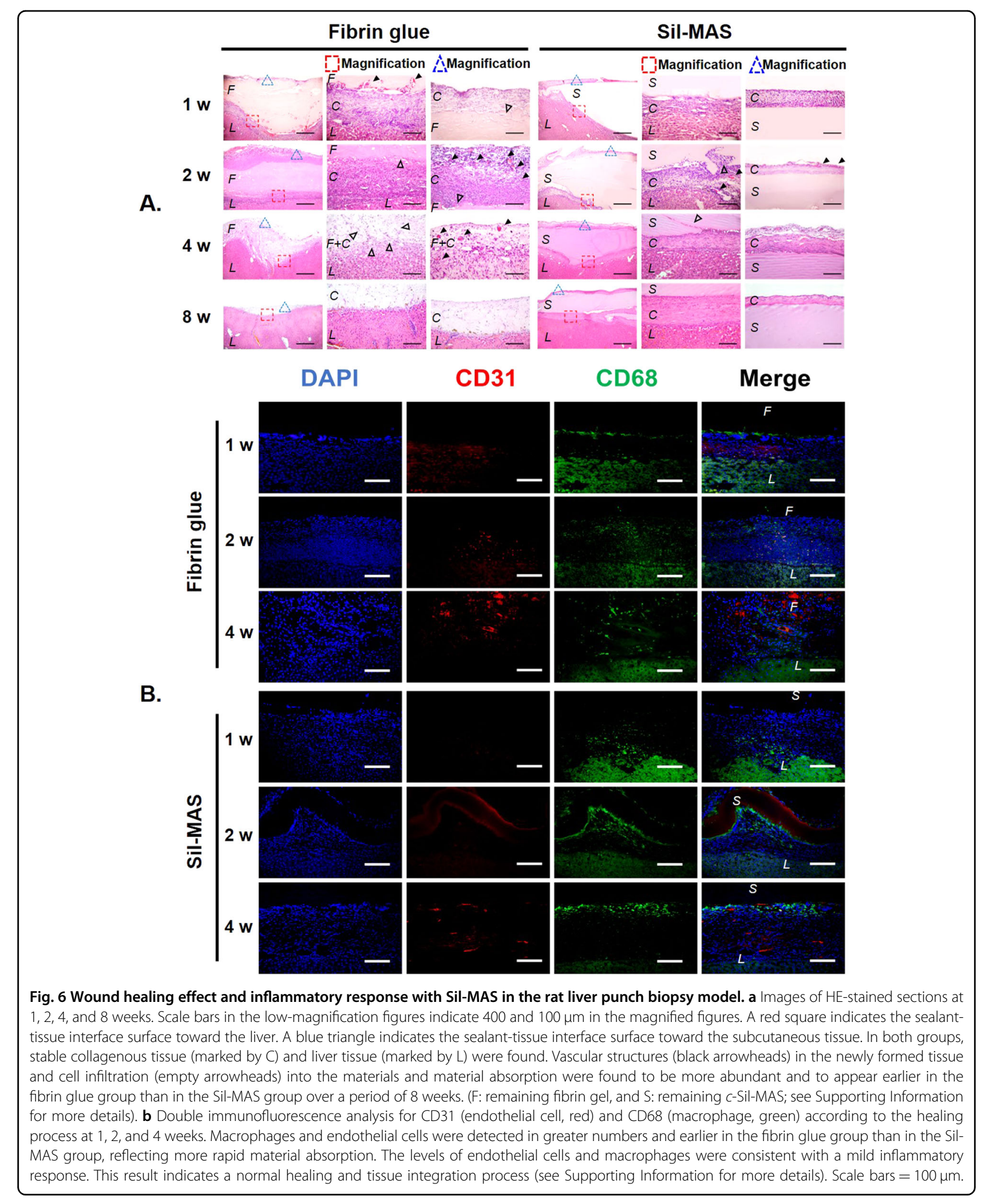


A.
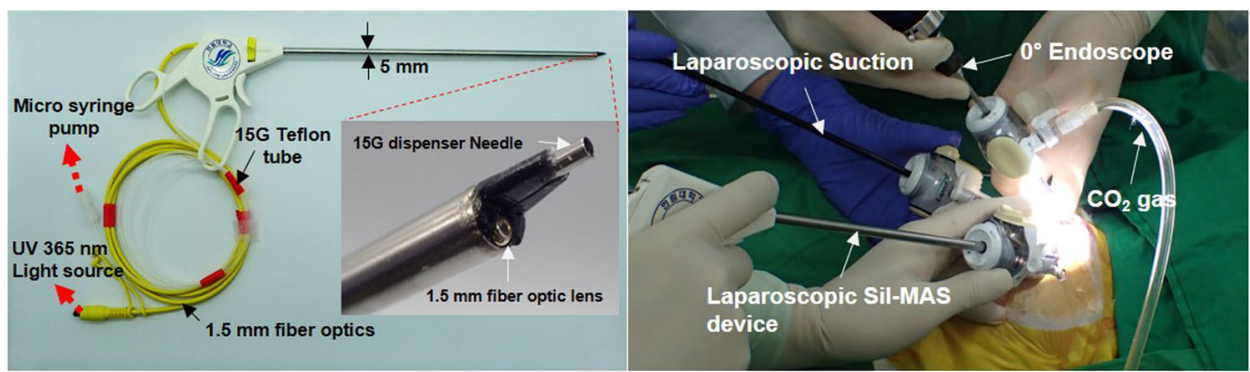

B.
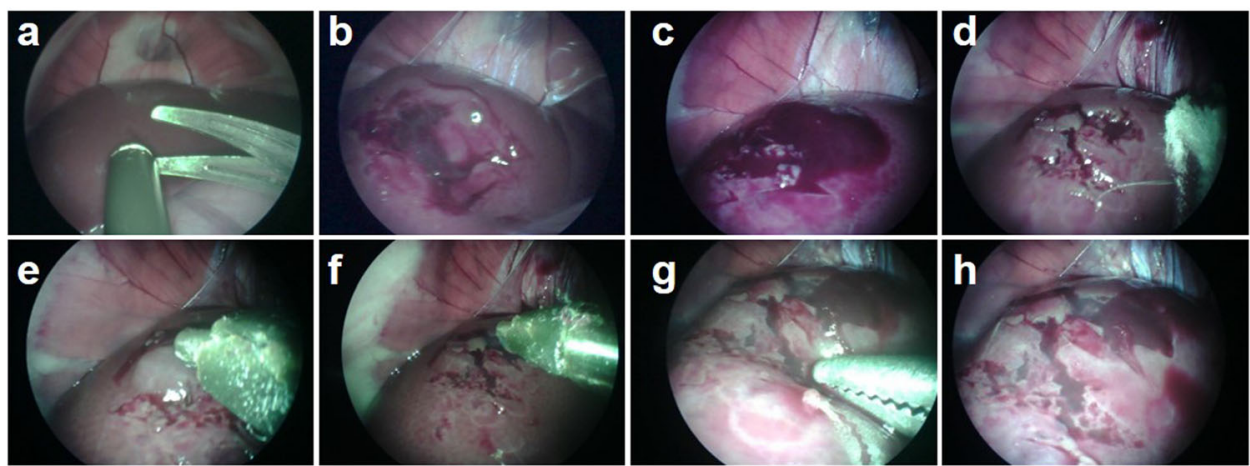

C.
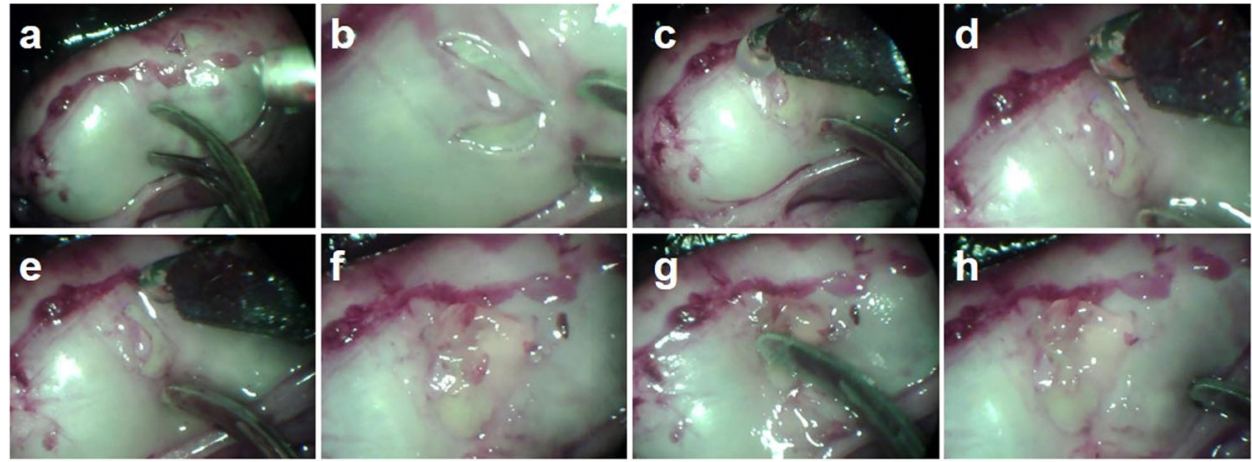

Fig. 7 Laparoscopic surgery using a homemade laparoscopic Sil-MAS device in a rabbit laceration model of liver and stomach serosa. a Photographs of a homemade laparoscopic Sil-MAS device and a three-pot laparoscopic operation field. $\mathbf{b}$ Laparoscopic surgical procedure images in the liver laceration model, $(a-b)$ making of the deep and superficial liver laceration using endoscopic scissors and suction, (c) bleeding and hematoma on the liver, (d) briefly pressing with gauze on bleeding liver laceration lesions, (e) applying I-Sil-MAS (1.0 cc) using the homemade laparoscopic Sil-MAS device, ( $f$ ) UV light (20 s) exposure through a fiber optic lens in the device, ( $g$ ) checking for rebleeding or leakage of blood at the lesions by endoscopic forceps, and (h) complete adhesion and gelation of I-Sil-MAS on the liver laceration lesion (Video S7). c Laparoscopic surgical procedure images in the laceration model of stomach serosa. $(a-b)$ Making a laceration lesion in stomach serosa using endoscopic scissors, $(c-d)$ applying I-Sil-MAS (1.0 cc) using a homemade laparoscopic Sil-MAS device on the laceration lesion of stomach serosa, (e) UV light (20 s) exposure through a fiber optic lens in the device, $(f-g)$ checking of the adhesiveness and detachment of serosa at the lesions by endoscopic forceps, and ( $h$ ) complete adhesion and gelation of I-Sil-MAS on the laceration lesion of stomach serosa (Video S8).

penetration of $l$-Sil-MAS into the irregular tissue surfaces and covalent bonding of the radicals generated during the photopolymerization process ${ }^{26}$. Additionally, the hydrophobic/hydrophilic interactions and electrostatic interactions between the tissue surface and $l$-Sil-MAS would increase adhesion. The adhesion mechanism of Sil-MAS needs to be further studied to improve our understanding.
The international commission on nonionizing radiation protection (ICNIRP) guidelines recommend limiting UV exposure at $365 \mathrm{~nm}$ to $27 \mathrm{~J} / \mathrm{cm}^{2}$ for the eyes and $\operatorname{skin}^{27}$. Accordingly, our condition $\left(0.18 \mathrm{~J} / \mathrm{cm}^{2}\right.$ at $\left.365 \mathrm{~nm}\right)$ is well within this limit. It has been reported that LAP, a type I photoinitiator, minimizes cytotoxicity compared with Irgacure $2959^{28}$. In addition, in contrast to Irgacure 2959, 
which has a low water solubility $(<0.5 \%)$ and a molar extinction coefficient of $4 \mathrm{M}^{-1} \mathrm{~cm}^{-1}$ at $365 \mathrm{~nm}^{28}$, LAP possesses higher water solubility $(<8.5 \%)$ and faster polymerization rates due to its higher molar extinction coefficient $\left(218 \mathrm{M}^{-1} \mathrm{~cm}^{-1}\right.$ at $\left.365 \mathrm{~nm}\right)$. If slowed gelation is acceptable, LAP also enables work in the safe visible light region $(>400 \mathrm{~nm})$ because of LAP's visible light absorption tail. Free radicals formed during photopolymerization can be more cytotoxic than the photoinitiator itself $f^{29}$; however, it is known that the lifetime of free radicals is shorter than a second and may be promptly quenched following light exposure ${ }^{29}$. Nevertheless, safety evaluations of longer periods of Sil-MAS treatment should be further conducted.

Many sealants are derived from living sources. Animalderived products such as collagen or gelatin involve high costs and the risk of pathogen transmission. Fibrin glue use in surgical procedures has gained popularity in recent years, and its efficacy in hemostasis is well known. However, fibrin glue has poor adhesive functions, difficulties of gelation control, and weak mechanical properties on wet tissues. Additionally, human-derived products risk transmitting viral infections (hepatitis or AIDS) or potentially prion disease. Recombinant products such as MeTro ${ }^{30}$ eliminate the risk of infectious transmission; however, they may cause allergic reactions secondary to the hamster or snake proteins used during processing. In contrast, $\mathrm{SF}$ is a superior biocompatible material that is nontoxic, biodegradable, and approved by the $\mathrm{FDA}^{4,12,15}$. Using published market data, the price per milligram (online search in 2018) of the starting materials for the various sealants is as follows: bovine type I collagen $(\$ 1.6 / \mathrm{mg})$, human recombinant tropoelastin $(\$ 333 / \mathrm{mg})$, human serum albumin $(\$ 175 / \mathrm{mg})$, and fibrinogen from human plasma $(\$ 1.1 / \mathrm{mg})$. For SF, the cost is only $\$ 0.29 / \mathrm{mg}$. Given all of these advantages, assuming FDA approval and competitive pricing of SF, we would expect Sil-MAS to be rapidly adopted for clinical practice, with low barriers to switching for surgeons.

\section{Conclusions}

We present, for the first time, the applicability of a photocurable SF sealant on both wet and dry soft tissue. Sil-MAS provided strong and long-lasting sealing through its excellent adhesive properties, hemostatic effects, and wound healing effect in vitro and in vivo. In addition, SilMAS showed biocompatibility and an adequate degradation time, which eliminated the need for retreat or removal of Sil-MAS from the wound site. In particular, the proof-of-concept studies using Sil-MAS and a developed laparoscopic tool suggest application prospects in the field of robotic surgery. Overall, our study suggests that Sil-MAS could be a versatile medical glue worth being applied in clinical use.

\section{Acknowledgements}

This work was supported by the National Research Foundation of Korea (NRF) grant funded by the Korea government (MSIT) (grant no. 2016R1E1A1A01942120), Cooperative Research Program for Agriculture Science and Technology Development (project no. PJ01313901) Rural Development Administration, Republic of Korea and the Hallym University research fund. We thank Dr. Harry Jung for assistance in double immunostaining.

\section{Author details}

${ }^{1}$ Nano-Bio Regenerative Medical Institute, College of Medicine, Hallym University, Chuncheon 24252, Republic of Korea. ${ }^{2}$ School of Medicine, George Washington University, Washington, DC 20037, USA. ${ }^{3}$ Departments of Otorhinolaryngology-Head and Neck Surgery, Chuncheon Sacred Heart Hospital, School of Medicine, Hallym University, Chuncheon 24252, Republic of Korea. ${ }^{4}$ Gordon Center for Medical Imaging, Department of Radiology, Massachusetts General Hospital and Harvard Medical School, Boston, MA 02114, USA. ${ }^{5}$ Departments of Neurosurgery, Chuncheon Sacred Heart Hospital, School of Medicine, Hallym University, Chuncheon 24253, Republic of Korea

\section{Author contributions}

S.H.K. and Y.J.L. designed the experimental groups and collected and analyzed the data. S.H.K. wrote the paper. Y.J.L. led all the experiments with contributions from D.Y.K., H.J.L., J.M.L., and M.T.S. J.R.C. assisted in editing the manuscript, and J.R.C. and Y.J.C. determined clinical applications from the results. J.S.L., O.J.L., H.H., H.L., O.A., and Y.J.S. supported the experimental setup. H.S.C. contributed to manuscript editing and provided scientific advice. C.H.P. conceived and supervised the overall research. All authors discussed the results and commented on the paper.

\section{Data availability}

The authors declare that all data supporting the results in this study are available within the paper and its Supporting Information.

Conflict of interest

The authors declare that they have no conflict of interest.

\section{Publisher's note}

Springer Nature remains neutral with regard to jurisdictional claims in published maps and institutional affiliations.

Supplementary information is available for this paper at https://doi.org/ 10.1038/s41427-020-0227-6.

Received: 17 October 2019 Revised: 3 April 2020 Accepted: 7 April 2020. Published online: 26 June 2020

\footnotetext{
References

1. Spotnitz, W. D. \& Burks, S. Hemostats, sealants, and adhesives: components of the surgical toolbox. Transfusion 48, 1502-1516 (2008).

2. Spotnitz, W. D. Fibrin sealant: the only approved hemostat, sealant, and adhesive-a laboratory and clinical perspective. ISRN surgery 2014 (2014).

3. Lee, O. J. et al. Fabrication and characterization of hydrocolloid dressing with silk fibroin nanoparticles for wound healing. Tissue Eng. Regen. Med. 13, 218-226 (2016)

4. Moon, B. M. et al. Novel fabrication method of the peritoneal dialysis filter using silk fibroin with urease fixation system. J. Biomed. Mater. Res. B 105 2136-2144 (2017)

5. Lee, J. H., Lee, J. S., Kim, D.-K, Park, C. H. \& Lee, H. R. Clinical outcomes of silk patch in acute tympanic membrane perforation. Clin. Exp. Otorhinolar 8, 117 (2015).

6. Seok, H. et al. Comparison of bio-degradation for ridge preservation using silk fibroin-based grafts and a collagen plug. Tissue Eng. Regen. Med. 14, 221-231 (2017).

7. Dal Pra, I., Freddi, G., Minic, J., Chiarini, A. \& Armato, U. De novo engineering of reticular connective tissue in vivo by silk fibroin nonwoven materials. Biomaterials 26, 1987-1999 (2005).
} 
8. Park, H. J. et al. Fabrication of $3 \mathrm{D}$ porous silk scaffolds by particulate (salt/ sucrose) leaching for bone tissue reconstruction. Int. J. Biol. Macromol. 78 215-223 (2015).

9. Moon, B. M. et al. The pilot study for the development of the hemostatic agent using silk fibroin for epistaxis. Tissue Eng. Regen. Med. 10, 41-47 (2013).

10. Seo, Y. B. et al. In vitro and in vivo evaluation of the duck's feet collagen sponge for hemostatic applications. J. Biomater. Appl. 32, 484-491 (2017).

11. Yang, Y. J. et al. Multifunctional adhesive silk fibroin with blending of RGDbioconjugated mussel adhesive protein. Biomacromolecules 15, 1390-1398 (2014).

12. Serban, M. A., Panilaitis, B. \& Kaplan, D. L. Silk fibroin and polyethylene glycolbased biocompatible tissue adhesives. J. Biomed. Mater. Res. A 98, 567-575 (2011).

13. Burke, K. A., Roberts, D. C. \& Kaplan, D. L. Silk fibroin aqueous-based adhesives inspired by mussel adhesive proteins. Biomacromolecules 17, 237-245 (2015).

14. Bai, S. et al. A silk-based sealant with tough adhesion for instant hemostasis of bleeding tissues. Nanoscale Horiz. 4, 1333-1341 (2019).

15. Kim, S. H. et al. Precisely printable and biocompatible silk fibroin bioink for digital light processing 3D printing. Nat. Commun. 9, 1620 (2018).

16. Bhardwaj, N. et al. Silk fibroin-keratin based 3D scaffolds as a dermal substitute for skin tissue engineering. Int. J. Integr. Biol. 7, 53-63 (2015).

17. Ghobril, C. \& Grinstaff, M. The chemistry and engineering of polymeric hydrogel adhesives for wound closure: a tutorial. Chem. Soc. Rev. 44 1820-1835 (2015).

18. Hope, W. W. et al. An evaluation of electrosurgical vessel-sealing devices in biliary tract surgery in a porcine model. HPB 12, 703-708 (2010).

19. Bao, P. et al. The role of vascular endothelial growth factor in wound healing. J. Surg. Res. 153, 347-358 (2009).

20. Lenselink, E. A. Role of fibronectin in normal wound healing. Int. Wound J. 12, 313-316 (2015).
21. Cheng, F. et al. Vimentin coordinates fibroblast proliferation and keratinocyte differentiation in wound healing via TGF- $\beta-S l u g$ signaling. Proc. Natl Acad. Sci. USA 113, E4320-E4327 (2016).

22. Park, Y. R. et al. NF-kB signaling is key in the wound healing processes of silk fibroin. Acta Biomater. 67, 183-195 (2018).

23. Kalucka, J., Bierhansl, L., Wielockx, B., Carmeliet, P. \& Eelen, G. Interaction of endothelial cells with macrophages-linking molecular and metabolic signaling. Pflügers Archiv-Eur. J. Appl. Physiol. 469, 473-483 (2017).

24. Emir, S. et al. The efficacy of fibrin glue to control hemorrhage from the gallbladder bed during laparoscopic cholecystectomy. Turk. J. Surg. ish J. Surg. Ulus. Cerrahi Derg. 29, 158 (2013).

25. Pisanu, A., Reccia, I., Porceddu, G. \& Uccheddu, A. Meta-analysis of prospective randomized studies comparing single-incision laparoscopic cholecystectomy (SILC) and conventional multiport laparoscopic cholecystectomy (CMLC). J. Gastrointest. Surg. 16, 1790-1801 (2012).

26. Lang, $\mathrm{N}$. et al. A blood-resistant surgical glue for minimally invasive repair of vessels and heart defects. Sci. Transl. Med. 6, 218ra216-218ra216 (2014).

27. International Commission on Non-lonizing Radiation Protection. et al. Guidelines on limits of exposure to ultraviolet radiation of wavelengths between $180 \mathrm{~nm}$ and $400 \mathrm{~nm}$ (incoherent optical radiation). Health Phys. 87, 171-186 (2004).

28. Fairbanks, B. D., Schwartz, M. P., Bowman, C. N. \& Anseth, K. S. Photoinitiated polymerization of PEG-diacrylate with lithium phenyl-2, 4, 6-trimethylbenzoylphosphinate: polymerization rate and cytocompatibility. Biomaterials 30, 6702-6707 (2009).

29. Xu, L., Sheybani, N., Yeudall, W. A. \& Yang, H. The effect of photoinitiators on intracellular AKT signaling pathway in tissue engineering application. Biomater Sci. 3, 250-255 (2015)

30. Annabi, N. et al. Engineering a highly elastic human protein-based sealant for surgical applications. Sci. Transl. Med. 9, eaai7466 (2017). 